\title{
Operationalisierung und Auswertung
}

\subsection{Der Fragebogen: Operationalisierung des Erkenntnisinteresses im Kontext von Theorie und Forschungsstand}

Die Operationalisierung der Forschungsleitfragen - die Übersetzung von abstrakten Begriffen in konkrete Forschungsoperationen - erfolgte vermittels der Formulierung von Fragen und Antwortkategorien in einem standardisierten Fragebogen, der im elektronischen Zusatzmaterial zu diesem Kapitel einzusehen ist. Standardisierung bedeutet, dass die Reihenfolge und der Wortlaut der Fragen und Antwortmöglichkeiten feststehen und zutreffende Antworten anzukreuzen oder vorgegebene Aussagen anhand einer Skala zu bewerten sind. Dafür wurden entsprechend des Erkenntnisinteresses, unter Berücksichtigung des Forschungsstandes (I. 2.2) und theoretischer Annahmen zu (Struktur-)Parametern, die das Organisationshandeln beeinflussen (II. 4.3), sechs zentrale Themenblöcke gebildet:

1. Allgemeine Merkmale der MSO

2. Ressourcenausstattung und Organisationsdemografie

3. Untersuchungsfokus: Freiwillig Engagierte

4. Ziele, Selbstverständnis, internes Miteinander

5. Aktivitäten und Dienstleistungen

6. Kooperations- und Umweltbeziehungen.

Elektronisches Zusatzmaterial Die elektronische Version dieses Kapitels enthält Zusatzmaterial, das berechtigten Benutzern zur Verfügung steht https://doi.org/10.1007/978-3-658-34298-2_6. 
Die mit empirischen Studien und den organisationssoziologischen Kernelementen ermittelten Einflussfaktoren, die in der Summe das Fundament für das tatsächliche Geschehen in einer Organisation darstellen, wurden in den jeweiligen Kapiteln bereits detailliert thematisiert. Die ihnen zugeordneten und zu operationalisierenden Aspekte werden im Folgenden zur Nachvollziehbarkeit als Kondensat in einer Tabelle aufgeführt und ihre Überführung in Fragen im Erhebungsbogen kenntlich gemacht (Tab. 6.1). In diesem Zusammenhang ist zum einen darauf hinzuweisen, dass die Bestimmungsfaktoren konzeptuell nicht überschneidungsfrei und trennscharf voneinander abzugrenzen sind. So wird etwa die finanzielle Ressourcenausstattung (intern) auch von Fördermaßnahmen (extern) beeinflusst. Das Selbstverständnis der MSO bestimmt organisationsspezifische Ziele und Aufgabenfelder wie auch das Kooperationsverhalten von MSO. Zum anderen wird deutlich, dass sich organisationstheoretische und empirisch fundierte Elemente in großen Teilen decken und in der Operationalisierung zusammenfließen.

Das der Organisationsanalyse zugrunde gelegte quantitative Paradigma, mit dem für eine zuvor bestimmte Grundgesamtheit von MSO Muster, verallgemeinerbare Aussagen und Zusammenhänge zwischen zwei oder mehreren Merkmalen gefunden und bestenfalls neue Erkenntnisse gewonnen werden sollen, basiert in Teilen auf der Formulierung von Hypothesen. Hypothesen stellen wissenschaftlich fundierte Vermutungen über Sachverhalte und Zusammenhänge auf und beinhalten damit gesichertes Wissen und unbestätigte Annahmen zugleich (Pollack 2014a: 405). Neben einer deskriptiven Bestandsaufnahme der organisationalen Performanzen und Ressourcenausstattungen stellt die Prüfung der theoriegeleiteten Hypothesen im Rahmen dieser Arbeit ein wesentliches forschungsleitendes Ziel dar. Die Möglichkeit ihrer Bestätigung oder Widerlegung ist mit dem Erhebungsinstrument sicherzustellen. Auf die Nachweiserbringung ihrer KontrollGewährleistung vermittels einer Zerlegung der Hypothesen in ihre einzelnen Bestandteile (Variablen) wird im Rahmen dieses Kapitels aber verzichtet.

Um einen möglichst komplett ausgefüllten Fragebogen zurückzuerhalten, Frustrationen und Abbrïche zu vermeiden, war es ein zentrales Ziel, den Erhebungsbogen mit einer Obergrenze von maximal zehn DIN A4-Seiten nicht zu umfangreich, inhaltlich verständlich und konsistent zu gestalten, d. h. die Fragen entsprechend der Reihenfolge der oben aufgeführten Themenblöcke anzuordnen. Inhaltlich war es eine wesentliche Herausforderung, einen Fragebogen zu konzipieren, der entsprechend der forschungsleitenden Fragen sowohl für religiöse als auch nicht-religiöse (,säkulare“) und darüber hinaus - im Sinne eines one-fitsall - für grundsätzlich heterogen aufgestellte MSO beantwortbar bzw. mit den ungleichen Organisationrealitäten inhaltlich kompatibel war. Zur Überprüfung der Eignung und Beantwortbarkeit der Fragen wurde mit zehn sehr unterschiedlich 
Tab.6.1 Theorie- und empiriebasierte Einflussfaktoren für organisationales Handeln

\begin{tabular}{|c|c|c|c|}
\hline $\begin{array}{l}\text { Interne } \\
\text { Einflussfaktoren } \\
\text { (Innere Verfasstheit/ } \\
\text { interne Bedingungen) }\end{array}$ & Determinanten & Ausdifferenzierung & $\begin{array}{l}\text { Operationa- } \\
\text { lisierung } \\
\text { (Fragennr./ } \\
\text { Fragebogen) }\end{array}$ \\
\hline Strukturell & $\begin{array}{l}\text { Finanzielle } \\
\text { und räumliche } \\
\text { Ausstattung }\end{array}$ & $\begin{array}{l}\text { - Finanzielle Ressourcen } \\
\text { - Zugang zu Räumlichkeiten }\end{array}$ & $\begin{array}{l}14,15 \\
16\end{array}$ \\
\hline \multirow[t]{2}{*}{$\begin{array}{l}\text { Strukturell/organisa- } \\
\text { tionsdemografisch }\end{array}$} & Mitgliederstruktur & $\begin{array}{l}\text { - Anzahl der Mitglieder } \\
\text { - Zusammensetzung/Sozialstruktur } \\
\text { - Heterogenität/Homogenität } \\
\text { - Demografischer Wandel/ } \\
\text { - Generationenkonflikte }\end{array}$ & $\begin{array}{l}7,25 \\
8,9,10 \\
8,9,10 \\
28\end{array}$ \\
\hline & $\begin{array}{l}\text { Personelle } \\
\text { Ausstattung }\end{array}$ & $\begin{array}{l}\text { - Anzahl von Hauptamtlichen } \\
\text { - Anzahl von Engagierten }\end{array}$ & $\begin{array}{l}17,18,25 \\
19,20,22,25\end{array}$ \\
\hline Ideell & $\begin{array}{l}\text { Selbstverständ- } \\
\text { nis/Ziele/Tätig- } \\
\text { keiten }\end{array}$ & $\left.\begin{array}{l}\text { - Religiöse Überzeugungen } \\
\text { - Migrationsspezifische Interessen } \\
\text { - Aufnahmelandorientierung } \\
\text { - Herkunftslandorientierung } \\
\text { - Binnenorientierung vs. Außen- } \\
\text { orientierung } \\
\text { - Migrationsgeschichte }\end{array}\right\}$ & $\begin{array}{l}5,6 \\
26,27,29 \\
30,31,34\end{array}$ \\
\hline Ideell/strukturell & $\begin{array}{l}\text { Umweltbezie- } \\
\text { hungen/Außen- } \\
\text { verhältnis }\end{array}$ & $\begin{array}{l}\text { - Haltung gegenüber Nicht- } \\
\text { Mitgliedern, Andersgläubigen, } \\
\text { außerreligiösen, politischen/ } \\
\text { staatlichen Akteuren } \\
\text { - Zugang zu Wissen und Informa- } \\
\text { tionen }\end{array}$ & $11,12,33$ \\
\hline $\begin{array}{l}\text { Externe } \\
\text { Einflussfaktoren } \\
\text { (Bedingungen und } \\
\text { Einflüsse aus der } \\
\text { Umwelt) }\end{array}$ & Determinanten & Ausdifferenzierung & $\begin{array}{l}\text { Operationa- } \\
\text { lisierung } \\
\text { (Fragennr./ } \\
\text { Fragebogen) }\end{array}$ \\
\hline Strukturell & Rechtsstatus & $\begin{array}{l}\text { - Eingetragener Verein (e. V.), } \\
\text { Körperschaft des öffentlichen } \\
\text { Rechts (KdöR) } \\
\text { - Mitgliedschaft im Dachverband }\end{array}$ & 3 \\
\hline Strukturell & $\begin{array}{l}\text { Anreize und } \\
\text { Gelegenheits- } \\
\text { strukturen }\end{array}$ & $\begin{array}{l}\text { - Finanzielle Förderungen } \\
\text { (staatliche Zuschüsse etc.) } \\
\text { - Politische Gelegenheitsstruktu- } \\
\text { ren/politische Orientierungen }\end{array}$ & $\begin{array}{l}13,32 \\
26,27,29 \\
31,32,36,37\end{array}$ \\
\hline Strukturell & $\begin{array}{l}\text { Organisationales } \\
\text { Feld }\end{array}$ & $\begin{array}{l}\text { - Ideelle, materielle, personelle } \\
\text { Unterstützung (z. B. durch } \\
\text { Kirchen, Wohlfahrtsverbände) } \\
\text { - Kontakte ins Herkunftsland } \\
\text { - Transnationale Beziehungen } \\
\text { (z.B. Austausch mit Diaspora- } \\
\text { Standorten) }\end{array}$ & $\begin{array}{l}26,29 \\
34,36\end{array}$ \\
\hline Ideell & $\begin{array}{l}\text { Öffentliche } \\
\text { Diskurse }\end{array}$ & $\begin{array}{l}\text { - Wahrnehmung öffentlicher } \\
\text { Diskurse }\end{array}$ & 40 \\
\hline
\end{tabular}


Tab.6.1 (Fortsetzung)

\begin{tabular}{|c|c|c|c|}
\hline $\begin{array}{l}\text { Relationale } \\
\text { Einflussfaktoren } \\
\text { (Beziehung zu } \\
\text { anderen Organisa- } \\
\text { tionen/Akteuren) }\end{array}$ & Determinanten & Ausdifferenzierung & $\begin{array}{l}\text { Operationa- } \\
\text { lisierung } \\
\text { (Fragennr./ } \\
\text { Fragebogen) }\end{array}$ \\
\hline Ideell & $\begin{array}{l}\text { Selbstverständ- } \\
\text { nis/Ziele/Tätig- } \\
\text { keiten (s.o.) }\end{array}$ & -s. oben & \\
\hline \multirow[t]{2}{*}{ Ideell/strukturell } & $\begin{array}{l}\text { Umweltbezie- } \\
\text { hungen/Außen- } \\
\text { verhältnis (s.o.) }\end{array}$ & $\begin{array}{l}\text { - s. oben } \\
\text { - Inhaltliche/finanzielle Beeinflus- } \\
\text { sung von außen }\end{array}$ & $13,32,37,39$ \\
\hline & $\begin{array}{l}\text { Zusammenarbeit } \\
\text { mit anderen Orga- } \\
\text { nisationen }\end{array}$ & $\begin{array}{l}\text { - Stellung der Akteure zueinander } \\
\text { - Motivation/Ziele } \\
\text { - Kommunikation } \\
\text { - Ressourcen } \\
\text { - Vertrauen } \\
\text { - Konkurrenz } \\
\text { - Konflikte } \\
\text { - Negativ-Zuschreibungen } \\
\text { - Bürokratische Barrieren }\end{array}$ & $32,35-39$ \\
\hline
\end{tabular}

ausgerichteten MSO ein Pretest durchgeführt. Dieser ermöglichte es, in Bezug auf die Formulierung von Ausfüllerläuterungen, Fragen und Antwortvorgaben auftretende Verständnisschwierigkeiten zu identifizieren, den Fragebogen daraufhin nochmal zu überarbeiten und mit sprachlichen und typografischen Mitteln (Fettsetzungen, Unterstreichungen, Pfeilen etc.) die Verständlichkeit und Qualität des Erhebungsinstrumentes zu verbessern.

An dieser Stelle soll nochmals darauf hingewiesen werden, dass die MSO in dieser Arbeit vermittels der Befragung von Funktionsträgern auf der Mesoebene und als Kollektivakteure untersucht werden. Um die schriftliche Beantwortung der Fragen gebeten wurden Vorstandsvorsitzende bzw. Personen, die mit ihrer Organisation sehr gut vertraut sind. Bei der Beantwortung einiger Fragen waren explizit Schätzungen erlaubt. Die Antworten beruhen folglich auch auf persönlichen Einschätzungen und subjektiv-selektiven Wahrnehmungen der Funktionsträger, die als Repräsentanten der MSO und Experten über ihr „Betriebswissen“ Auskunft geben. Es handelt sich somit um durch die Person eines Funktionsträgers gefilterte und kommunizierte Informationen zu organisationalen Eigenschaften. Diese Filterfunktion und das Hervortreten des Relevanzsystems des Funktionsträgers, der Aussagen für die Mitglieder trifft, müssen bei der Betrachtung der Ergebnisse stets mitgedacht werden. Letztendlich können MSO als Kollektivakteure, die sich aus Individuen mit je eigenen Interessen und Präferenzen zusammensetzen, nicht 
personifiziert werden. Eine alternativ in Erwägung zu ziehende Mitgliederbefragung in den Organisationen wäre aufgrund einer mangelnden finanziellen und personellen Ausstattung nicht durchführbar gewesen. Selbst beim Vorhandensein eines ganzen Forschungsteams ist eine solche aufgrund eines sich grundsätzlich schwierig gestaltenden Zugangs zu den Organisationen bzw. ihren Mitgliedern nur mit großen Anstrengungen zu realisieren (s. dazu exemplarisch Klöckner 2016: $221 \mathrm{ff}$.).

Im Folgenden wird komprimiert auf die Dramaturgie des Fragebogens, d. h. die Sukzession der Fragen und Antwortvorgaben, deren Inhalte und Formen eingegangen. Mit der Form ist die Unterscheidung der Fragen in geschlossene, halboffene und offene Fragen gemeint (Porst 2014: 53 ff.). ${ }^{1}$ Auf die Arten der Fragen wie auch die Verwendung bestimmter Skalen wird im Folgenden nicht näher eingegangen. Stattdessen sind sie in den einzelnen jeden Themenblock abschließenden Tabellen vermerkt. An dieser Stelle soll lediglich darauf hingewiesen werden, dass bei der Konzipierung des Fragebogens wiederholt auf eine von links nach rechts invertiert codierte vierstufige ordinale Antwortskala zurückgegriffen wurde (,Trifft voll und ganz zu“ [Code 1], ,Trifft eher zu“ [Code 2],

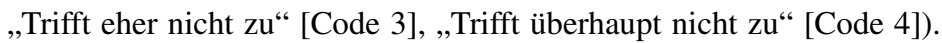

Mit Blick auf die Inhalte ist ebenfalls vorab herauszustellen, dass die Fragen überwiegend selbst konzipiert wurden. Zum Teil erfolgte aber auch eine Übernahme aus anderen Fragebögen oder eine Modifizierung des Wortlauts. Orientiert wurde sich an Fragen und Antwortkategorien aus organisations- und personenbezogenen Studien, die für eine schnelle Identifikation und Zuordnung innerhalb dieses Kapitels mit Kurztiteln versehen wurden (nachfolgend in eckigen Klammern). Konkret handelt es sich um folgende Studien: ZiviZ-Survey 2012 [ZiviZ]

\footnotetext{
${ }^{1}$ Während geschlossene Fragen eine bestimmte Anzahl von Antwortkategorien vorgeben, gibt es bei offenen gar keine: Über Linien wird der befragten Person signalisiert, dass die Antwort vollständig selbst anzugeben bzw. zu formulieren ist. Diese Form bietet sich bei Antizipation einer Vielfalt möglicher Antworten an (wie z. B. in Hinblick auf Gründungsjahre), da diese nicht in adäquater und effizienter Weise vorgegeben werden können. Bei ihrer Auswertung besteht jedoch ein höherer Aufwand, da die Antworten inhaltlich kategorisiert werden müssen. Bei halboffenen Fragen werden aufgeführte Antwortkategorien durch eine zusätzliche Kategorie „Sonstiges, und zwar: _ “ergänzt, womit die Möglichkeit der Formulierung einer eigenen Antwort besteht. Die halboffene Frage bietet sich an, wenn das Spektrum möglicher Antworten nur eingeschätzt, aber nicht vollständig abgesteckt werden kann. Zudem kann es vorkommen, dass sich die befragte Person mit den vorgegebenen Antwortkategorien auf eine geschlossene Frage ggf. nicht identifizieren kann. Die Berücksichtigung einer näher zu bestimmenden Restkategorie dient somit v. a. auch der Aufrechterhaltung der Motivation der Befragungsperson (Porst 2014: 59).
} 
(Krimmer/Priemer 2013); Solidarität mit Osteuropa: Praxis und Selbstverständnis christlicher Mittel- und Osteuropagruppen [Solidarität] (Gabriel et al. 2002); Migranten(dach)organisationen in Deutschland [MDO] (IntB 2011); Freiwilliges Engagement von Türkinnen und Türken in Deutschland [Türken] (Halm/Sauer 2005); Ehrenamtliche in der Flüchtlingsarbeit (EFA-Studie 2): Strukturen und Motive der ehrenamtlichen Flüchtlingsarbeit in Deutschland [EFA 2] (Karakayali/Kleist 2016); Motive des bürgerschaftlichen Engagements [Motive] (IfD Allensbach 2013) sowie das Kapitel „Anstoß und Motive für das freiwillige Engagement" aus dem Freiwilligensurvey 2014 [FWS] (Müller et al. 2016).

Zum Abschluss jedes Themenblocks und den darunter gebündelten Fragen wird in den Tabellen kenntlich gemacht, an welchen der genannten Studien (Kurztitel) sich hinsichtlich der Auswahl von konkreten Fragen und Antwortkategorien orientiert wurde, wo eine komplette Übernahme oder eine Modifikation erfolgte. Überwiegend zurückgegriffen wurde auf den Erhebungsbogen des ZiviZ-Surveys 2012.

\section{Themenblock 1: Allgemeine Merkmale der MSO}

Abgefragt wurden zunächst ganz basale Informationen, die die MSO charakterisieren: Die Stadt, in der die MSO ansässig ist, ihr Gründungsjahr, ihre Rechtsform sowie die Zugehörigkeit zu einem Dachverband, der zu spezifizieren war. Die Beantwortung der Frage nach der Zuordnung zu einer bestimmten religiösen Tradition oder Lehre (nein/ja) war hinsichtlich der forschungsleitenden Fragen und dem damit unmittelbar verknüpften dichotomischen Analysezugriff (religiös versus säkular) eine zentrale. Diese analytische Differenzierung entspricht nach Auffassung der Autorin einer übergeordneten, trennscharfen und objektiven Unterscheidungskategorie, die sich auf die Strukturebene der MSO als Kollektivakteure bezieht. Bei Bejahung sollte diese Tradition bzw. Lehre möglichst genau angegeben werden, denn für die tiefergehende Analyse der Wirkungsweise des religiösen Faktors auf die zivilgesellschaftlichen Performanzen ist es wichtig, eine spezifische Zuordnung der untersuchten MSO zu bestimmten Glaubensrichtungen vornehmen zu können. Die Frage fungierte als Filter, indem die Antwortkategorie „Nein“ mit dem Hinweis gekennzeichnet war, dass bei ihrer Markierung die darauffolgende Frage zu überspringen ist. ${ }^{2}$ Diese erkundigte sich danach, ob es ein Hauptanliegen der Organisation ist, die religiöse Tradition bzw. Lehre gemeinsam zu pflegen und nach

\footnotetext{
${ }^{2}$ Bei der Beantwortung der Fragen im Fragebogenprogramm Unipark wurden bei Markierung betreffender Antwortkategorien und der einhergehenden Erfüllung von Filterbedingungen aufgrund der eingestellten Filterführung für die befragten Funktionsträger bzw. ihre Organisationen irrelevante Fragen gar nicht erst angezeigt.
} 
außen zu verkünden. Sie wurde in Anlehnung an die konsensfähige und dominierende Definition des Begriffs der Religionsgemeinschaft von Gerhard Anschütz (Anschütz 1933, Art. 137, S. 633) bzw. der auf dieser basierenden des Bundesverwaltungsgerichts formuliert. Letztere lautet: „Unter Religionsgemeinschaft ist ein Verband zu verstehen, der die Angehörigen ein und desselben Glaubensbekenntnisses oder mehrerer verwandter Glaubensbekenntnisse zu allseitiger Erfüllung der durch das gemeinsame Bekenntnis gestellten Aufgaben zusammenfasst“ (BVerwG NJW 2005, 2101; 2102 m. w. N.).

Unabhängig von öffentlichen und wissenschaftlichen Debatten (u. a. Muckel 2017) fungierte im Rahmen dieser Studie also die (Nicht-)Zuordnung zu einer religiösen Lehre oder Tradition als Ausgangspunkt für die Einteilung der MSO in „,säkulare“ oder „religiöse“. Ebenfalls in Erfahrung gebracht werden sollte, ob sich eine religiöse Vereinigung als eine Religionsgemeinschaft mit einem umfassenden religiösen Selbstverständnis, einem Verkündigungsanliegen und umfangreichen religiösen Zielsetzungen versteht.

\begin{tabular}{|c|c|c|c|}
\hline \multicolumn{4}{|c|}{ - Themenblock 1: Allgemeine Merkmale der MSO } \\
\hline Nr. & Frage & Form der Frage & $\begin{array}{l}\text { Orientierung der Fragen }(\mathrm{F}) / \\
\text { Antwortvorgaben }(\mathrm{A}) \\
\text { an anderen Studien } \\
\text { (Nr./Seite) }\end{array}$ \\
\hline 1 & Stadt & offen & $-(=$ selbst konzipiert $)$ \\
\hline 2 & $\begin{array}{l}\text { Gründungsjahr } \\
\text { („Alter“ der Organisation) }\end{array}$ & offen & $\begin{array}{l}\text { F: ZiviZ, Nr. 1, S. } 86 \\
\text { (modifiziert) }\end{array}$ \\
\hline 3 & Rechtsform & Einfachnennung; halboffen & $\begin{array}{l}\text { F: ZiviZ, Nr. 4, S. } 86 \\
\text { (übernommen) } \\
\text { A: modifiziert }\end{array}$ \\
\hline 4 & $\begin{array}{l}\text { Mitglied in einem Dach- } \\
\text { verband }\end{array}$ & $\begin{array}{l}\text { Einfachnennung (nein/ja); } \\
\text { halboffen }\end{array}$ & $\begin{array}{l}\text { F: ZiviZ, Nr. 8, S. } 87 \\
\text { (modifiziert) } \\
\text { A: modifiziert }\end{array}$ \\
\hline 5 & $\begin{array}{l}\text { Zuordnung zu einer } \\
\text { bestimmten religiösen Tradi- } \\
\text { tion oder Lehre }\end{array}$ & $\begin{array}{l}\text { Einfachnennung (nein/ja); } \\
\text { halboffen; } \\
\text { Filter (nein: } \rightarrow \text { Frage } 7 \text { ) }\end{array}$ & - \\
\hline 6 & $\begin{array}{l}\text { Ob Hauptanliegen, religiöse } \\
\text { Tradition/Lehre gemeinsam } \\
\text { zu pflegen und nach außen } \\
\text { zu verkünden }\end{array}$ & $\begin{array}{l}\text { Einfachnennung (nein/ja); } \\
\text { geschlossen }\end{array}$ & - \\
\hline
\end{tabular}

\section{Themenblock 2: Ressourcenausstattung und Organisationsdemografie}

Bei der Beantwortung der Frage nach der Mitgliederzahl durfte explizit geschätzt werden. Bei der Auswertung erwiesen sich die gewählten Antwortvorgaben (,,unter 10“; ,,11 bis 50“; ,,51 bis 100“; ,, mehr als 100 Mitglieder, und zwar: _ “) als zu grob 
und nicht sinnvoll, da die meisten befragten Organisationen über 100 Mitglieder angaben und eine offene Frageform erkenntnisreichere Ergebnisse geliefert hätte.

In der darauffolgenden Frage wurden die Funktionsträger der MSO gebeten, alle Mitglieder ihrer Organisation mit einer sechsstufigen Antwortskala den fünf Personenkreisen „Berufstätige/Selbstständige“, ,Schüler und Studierende“, „Hausfrauen/Hausmänner“, „Arbeitslose/Arbeitssuchende“ oder „Rentner“ zuzuordnen. Damit sollte in stark vereinfachter Form über den beruflichen bzw. sozialen Status (Erwerbsstatus) der Mitglieder deren soziale Zusammensetzung ermittelt werden. Darüber hinaus wurden die überwiegenden Herkunftsländer der Mitglieder abgefragt. Bei den möglichen Mehrfachantworten wurden neun Länder gelistet, mit der Kategorie „Sonstiges“ konnten nicht erwähnte hinzugefügt werden.

Die sich der Frage nach den Herkunftsländern anschließende Bitte um Einschätzung der Zugehörigkeit der Mitglieder zu bestimmten Glaubensrichtungen stellte eine unkonventionelle Abfrageweise und sicherlich auch eine Herausforderung für die befragten Funktionsträger dar - insbesondere auch für diejenigen nicht-religiöser und mitgliederstarker MSO. In der Itembatterie vorgegeben waren neben den Kategorien „konfessionslos“ sowie „Sonstiges“ 15 Konfessionen, die insgesamt die Binnenpluralität des Christentums, des Islam (Alevitentums), des Judentums, den Buddhismus und Hinduismus sowie das Ezidentum berïcksichtigten. Geantwortet werden sollte wieder anhand einer sechsstufigen Skala. In einer Ausfüllerläuterung war vermerkt, dass nicht in jeder Zeile, sondern nur bei den in der MSO vertretenen Glaubensrichtungen Markierungen gesetzt werden sollten. Die Zuordnungen der Mitglieder zu den vorgegebenen Personenkreisen und Glaubensrichtungen funktionierten erstaunlich gut (s. auch 7.1.2.1).

Das mit den Fragen zu den Personenkreisen, Herkunftsländern und Konfessionszugehörigkeiten der Mitglieder verknüpfte Anliegen war zum einen, homogene oder heterogene, Mehr- oder Minderheits- oder ausbalancierte - paritätische - Verhältnisse festzustellen. Aus theoretischer Perspektive kann eine sozioökonomisch, ethnisch oder religiös homogene Mitgliederstruktur als Indiz für eine hohe Werthomogenität fungieren, wie sie insbesondere bei religiösen Vereinigungen vorzufinden ist (II. 4.2.4).

Die Tatsache, dass Organisationen bestimmte Konditionen und Voraussetzungen für Mitgliedschaften festlegen und so über Ein- und Austritte von Personen entscheiden können, wurde mit einer Filterfrage, ob es bestimmte Mitgliedschaftsbedingungen gibt (nein/ja), und einer darauffolgenden Frage nach den konkreten Bedingungen berücksichtigt. Die Beantwortung gibt Auskunft über Grenzziehungen bzw. die Aufgeschlossenheit einer Organisation. Von fünf, mehrheitlich aus 
dem ZiviZ-Survey 2012 übernommenen Antwortmöglichkeiten konnten mehrere markiert werden. ${ }^{3}$

Themenblock 2: Organisationsdemografie und Ressourcenausstattung

Mitglieder(-struktur)

\begin{tabular}{|c|c|c|c|}
\hline Nr. & Frage & Form der Frage & $\begin{array}{l}\text { Orientierung der Fragen }(\mathrm{F}) / \\
\text { Antwortvorgaben }(\mathrm{A}) \\
\text { an anderen Studien } \\
\text { (Nr./Seite) }\end{array}$ \\
\hline 7 & $\begin{array}{l}\text { Anzahl Mitglieder insgesamt } \\
\text { (Schätzung) }\end{array}$ & $\begin{array}{l}\text { Einfachnennung; halboffen } \\
\text { (mehr als 100, } \\
\text { und zwar: _) }\end{array}$ & $\begin{array}{l}\text { F: Solidarität, Nr. } 4 \text { (o.S.) } \\
\text { (modifiziert) } \\
\text { A: modifiziert }\end{array}$ \\
\hline 8 & $\begin{array}{l}\text { Personenkreise (beruflicher } \\
\text { Stand) (Schätzung) }\end{array}$ & $\begin{array}{l}\text { Mehrfachnennung; } \\
\text { geschlossen; } \\
\text { 6er-Skala (alle; mehr als } \\
\text { die Hälfte; ungefähr die } \\
\text { Hälfte; weniger als die } \\
\text { Hälfte; keine; weiß nicht) }\end{array}$ & - \\
\hline 9 & Herkunftsländer & $\begin{array}{l}\text { Mehrfachnennung; } \\
\text { halboffen }\end{array}$ & - \\
\hline 10 & $\begin{array}{l}\text { Zugehörigkeit der Mitglieder } \\
\text { zu bestimmten religiösen } \\
\text { Gemeinschaften (Schätzung) }\end{array}$ & $\begin{array}{l}\text { Mehrfachnennung; } \\
\text { halboffen; 6er-Skala (s.o.) }\end{array}$ & - \\
\hline 11 & $\begin{array}{l}\text { Ob Voraussetzungen für } \\
\text { Mitgliedschaft }\end{array}$ & $\begin{array}{l}\text { Einfachnennung (nein/ja); } \\
\text { Filter (nein: } \rightarrow \text { Frage 13) }\end{array}$ & $\begin{array}{l}\text { F: ZiviZ, Nr. 24, S. } 90 \\
\text { (übernommen) } \\
\text { A: modifiziert }\end{array}$ \\
\hline 12 & $\begin{array}{l}\text { Konkrete Voraussetzungen } \\
\text { für Mitgliedschaft }\end{array}$ & $\begin{array}{l}\text { Mehrfachnennung; } \\
\text { halboffen }\end{array}$ & $\begin{array}{l}\text { A: ZiviZ, Nr. 24, S. } 90 \\
\text { (modifiziert) }\end{array}$ \\
\hline
\end{tabular}

Da die finanzielle, räumlich-sachliche und personelle Ausstattung die Handlungsfähigkeit einer Organisation mitbestimmt, bezogen sich die nächsten drei Fragen auf die finanzielle Ausstattung der MSO. Gefragt wurde nach dominanten Finanzierungsquellen, von denen neun vorgegeben waren und mehrere angekreuzt werden konnten. Das jährlich zur Verfügung stehende Finanzvolumen war anhand sieben vorgegebener Items anzukreuzen und darüber hinaus auch die Existenz von finanziellen Engpässen (nein/ja) anzugeben.

Im Anschluss daran wurde gefragt, ob der Organisation Räumlichkeiten und hauptamtliche, bezahlte Mitarbeiter zur Verfügung stehen (nein/ja). Die Frage nach den Hauptamtlichen war als Filter konzipiert; bei ihrer Bejahung wurde sich mit

${ }^{3}$ Die Antwortvorgaben lauteten: „Empfehlungen von Mitgliedern“; „Die Zugehörigkeit $\mathrm{zu}$ einer bestimmten Glaubensgemeinschaft“; „Ein bestimmtes Alter (z. B. Mindestalter)“; ,Ein bestimmtes Geschlecht“; ,Ein bestimmter kultureller Hintergrund/eine bestimmte Nationalität“; , Sonstiges, und zwar: __“. 
einer offenen Angabe nach ihrer konkreten Anzahl erkundigt, wobei hier wieder explizit geschätzt werden durfte.

\begin{tabular}{|c|c|c|c|}
\hline \multicolumn{4}{|c|}{$\begin{array}{l}\text { Themenblock 2: Organisationsdemografie und Ressourcenausstattung } \\
\text { Finanzielle Ressourcen/Räumlichkeiten }\end{array}$} \\
\hline Nr. & Frage & Form der Frage & $\begin{array}{l}\text { Orientierung der Fragen }(F) \text { / } \\
\text { Antwortvorgaben }(A) \\
\text { an anderen Studien } \\
\text { (Nr./Seite) }\end{array}$ \\
\hline 13 & $\begin{array}{l}\text { Finanzierungsquellen der } \\
\text { Organisation (überwiegend) }\end{array}$ & $\begin{array}{l}\text { Mehrfachnennung; } \\
\text { halboffen }\end{array}$ & $\begin{array}{l}\text { F: Solidarität, Nr. } 38 \text { (o.S.) } \\
\text { (modifiziert); } \\
\text { A: modifiziert }\end{array}$ \\
\hline 14 & $\begin{array}{l}\text { Verfügbares Finanzvolumen } \\
\text { pro Jahr }\end{array}$ & Einfachnennung; halboffen & - \\
\hline 15 & Finanzielle Engpässe & $\begin{array}{l}\text { Einfachnennung (nein/ja); } \\
\text { geschlossen }\end{array}$ & $\begin{array}{l}\text { F: ZiviZ, Nr. 45, S. } 94 \\
\text { (modifiziert) } \\
\text { A: modifiziert }\end{array}$ \\
\hline 16 & $\begin{array}{l}\text { Verfügbarkeit von Räumlich- } \\
\text { keiten }\end{array}$ & $\begin{array}{l}\text { Einfachnennung (nein/ja); } \\
\text { geschlossen }\end{array}$ & $\begin{array}{l}\text { F: MDO, Nr. III. 1, S. } 92 \\
\text { (modifiziert) }\end{array}$ \\
\hline \multicolumn{4}{|c|}{ Hauptamtliche Mitarbeiter } \\
\hline 17 & $\begin{array}{l}\text { Ob Existenz hauptamtlicher, } \\
\text { bezahlter Mitarbeiter }\end{array}$ & $\begin{array}{l}\text { Einfachnennung (nein/ja); } \\
\text { Geschlossen; } \\
\text { Filter (nein: } \rightarrow \text { Frage 19) }\end{array}$ & $\begin{array}{l}\text { F: ZiviZ, Nr. 31, S. } 91 \\
\text { (modifiziert) } \\
\text { A: modifiziert }\end{array}$ \\
\hline 18 & $\begin{array}{l}\text { Anzahl insgesamt } \\
\text { (Schätzung) }\end{array}$ & offen & $\begin{array}{l}\text { F: ZiviZ, Nr. 32, S. } 92 \\
\text { (modifiziert) } \\
\text { A: modifiziert }\end{array}$ \\
\hline
\end{tabular}

\section{Themenblock 3: Untersuchungsfokus ,Freiwillig Engagierte}

Der Informationseinholung über die Existenz und Anzahl hauptamtlichen Personals schlossen sich insgesamt sechs Fragen nach in den MSO tätigen freiwillig Engagierten an. Allerdings wurden die Begriffe „Engagement“ oder „Engagierte“ im Fragebogen konsequent vermieden, um falschen oder Missverständnissen vorzubeugen, denn die Termini werden von Menschen mit Zuwanderungsgeschichte seltener verwendet und in anderen Sprachen fehlen Begriffsäquivalente (BMFSFJ 2017: 195).

In Analogie zur Erkundigung nach den Hauptamtlichen wurde nach dem Vorhandensein und der Anzahl der Personen, ,, die freiwillig UND unbezahlt bestimmte Aufgaben“ in der Organisation übernehmen, gefragt. Die Frage nach deren Existenz (nein/ja) fungierte als Filter: Wurde sie verneint, waren die folgenden sich auf die Freiwilligen beziehenden fünf Fragen zu überspringen und erst wieder die Frage Nr. 25 zu beantworten. 
In Übereinstimmung mit der Frage bei den Mitgliedern wurde auch bei den freiwillig Engagierten (zwecks Vergleichsanstellung) nach den unter diesen vertretenen fünf Personenkreisen ${ }^{4}$ gefragt. Um die Gesamtsituation hinsichtlich der Ausstattung mit und Rekrutierung von Freiwilligen beurteilen zu können, wurde des Weiteren zum einen um die Einschätzung von drei Aussagen zu den Engagierten gebeten. ${ }^{5}$ Mit einer darauffolgenden Frage wurde gezielt nach relevanten und bewusst verfolgten Mobilisierungs- bzw. Rekrutierungsstrategien gefragt. Mit Blick auf die Annahme von für religiöse und nicht-religiöse MSO hinsichtlich der Gewinnung von Freiwilligen unterschiedlich bedeutsamen Aspekten und der Überprüfung diesbezüglich theoretisch hergeleiteter Hypothesen (II. 4.4.2) wurden bei der Auswahl und Formulierung der acht Items auf persönliche interne Gegebenheiten und Beziehungsstrukturen sowie eher unpersönliche, extraorganisationale Opportunitäten und Akquise-Strategien abgehoben.

In einer weiteren Frage waren seitens der Funktionsträger die Motive, von denen sich die Engagierten im Rahmen ihrer Mitwirkung am stärksten leiten lassen, einzustufen. Mit dieser Vorgehensweise wurden die Engagierten also nicht direkt hinsichtlich ihrer individuellen Beweggründe befragt, was als unüblich zu werten ist. Diesbezüglich orientiert wurde sich an der Studie Solidarität mit Osteuropa (Gabriel et al. 2002). Nach ihrer Relevanz für die in den MSO freiwillig Engagierten einzuschätzen waren 13 Motive. Bei ihrer Auswahl als Items zu beachten war, dass sie nicht zu speziell und persönlich ausfallen, sondern für die Funktionsträger einschätzbar waren. Darüber hinaus mussten sie inhaltlich die Prüfung der Doppelhypothese mit Aussagen zu in religiösen und säkularen MSO jeweils bedeutsameren Motiven gewährleisten. Für die konkrete Auswahl der Motive als Hilfsmittel herangezogen wurde eine Studie, in der mit 24 unterschiedlichen Motiven eine Explorative Faktorenanalyse (EFA) durchgeführt wurde (IfD Allensbach 2013: 35 f., 74 ff.). Die für die Erhebung der vorliegenden Studie selektierten 13 Motive lassen sich in Anlehnung an diese EFA folgenden Komponenten zuordnen: Engagement, um 1.) Dinge zu verbessern und zu bewegen, 2.) um Sinngebung durch eine bedeutsame Aufgabe oder Anerkennung zu erfahren und um 3.) das eigene Leben durch Kontakte und Freude anzureichern. Zudem kann das Engagement 4.) mit (religiösen) Wertüberzeugungen und 5.) einem konkreten Nutzen

4 „Berufstätige/Selbstständige“, „Schüler und Studierende“, „Hausfrauen/Hausmänner“, „Arbeitslose/Arbeitssuchende“, „Rentner“.

${ }^{5} \mathrm{Zu}$ bewerten waren folgende drei Aussagen: „Wir können genügend Freiwillige gewinnen“; ,Für freiwillig und unbezahlt ausgeführte Leitungspositionen und Ämter (,Ehrenämter“) finden wir genug Freiwillige“; „Die Freiwilligen sind vorwiegend an zeitlich befristeten Tätigkeiten interessiert“". 
wie dem Erwerb von Qualifikationen oder dem Erhalt von Gegenleistungen verknüpft sein. Die Orientierung an dieser groben Kategorisierung ermöglichte es, die durch die Funktionsträger eingeschätzten Engagementmotive einzuhegen und grobe Stoßrichtungen auszumachen. Grundsätzlich ist die Tatsache, dass die Abfrage der Engagementmotive durch die Taxierungen der Funktionsträger erfolgte, kritisch zu reflektieren (s. dazu II. 4.3.3.2 und 7.1.2.4).

Die den Themenblock abschließende Frage richtete sich wieder an alle MSO und fokussierte nicht nur die Entwicklung der Zahlen von Engagierten, sondern auch von Mitgliedern und Hauptamtlichen in den letzten fünf Jahren (seit 2013). Sie ist in fast identischer Form im ZiviZ-Survey $2012 \mathrm{zu}$ finden.

Themenblock 3: Untersuchungsfokus „Freiwillig Engagierte“

\begin{tabular}{|c|c|c|c|}
\hline Nr. & Frage & Form der Frage & $\begin{array}{l}\text { Orientierung der Fragen (F)/ } \\
\text { Antwortvorgaben (A) } \\
\text { an anderen Studien } \\
\text { (Nr./Seite) }\end{array}$ \\
\hline 19 & $\begin{array}{l}\text { Ob Existenz von Personen, } \\
\text { die freiwillig UND unbezahlt } \\
\text { bestimmte Aufgaben über- } \\
\text { nehmen }\end{array}$ & $\begin{array}{l}\text { Einfachnennung (nein/ja); } \\
\text { geschlossen; } \\
\text { Filter (nein: } \rightarrow \text { Frage 25) }\end{array}$ & $\begin{array}{l}\text { F: ZiviZ, Nr. 25, S. } 90 \\
\text { (modifiziert) } \\
\text { A: modifiziert }\end{array}$ \\
\hline 20 & $\begin{array}{l}\text { Anzahl insgesamt } \\
\text { (Schätzung) }\end{array}$ & offen & - \\
\hline 21 & $\begin{array}{l}\text { Personenkreise (beruflicher } \\
\text { Stand) (Schätzung) }\end{array}$ & $\begin{array}{l}\text { Geschlossen; 6er-Skala } \\
\text { (alle; mehr als die Hälfte; } \\
\text { ungefähr die Hälfte; weni- } \\
\text { ger als die Hälfte; keine; } \\
\text { weiß nicht) }\end{array}$ & - \\
\hline 22 & $\begin{array}{l}\text { Einschätzung von Aussagen } \\
\text { zu Engagierten }\end{array}$ & $\begin{array}{l}\text { 4er-Skala (Trifft voll und } \\
\text { ganz zu ... Trifft überhaupt } \\
\text { nicht zu); geschlossen }\end{array}$ & $\begin{array}{l}\text { F: ZiviZ, Nr. 30, S. } 91 \\
\text { (modifiziert) } \\
\text { A: modifiziert }\end{array}$ \\
\hline 23 & $\begin{array}{l}\text { Mobilisierung von Enga- } \\
\text { gierten }\end{array}$ & 4er-Skala (s.o.); halboffen & - \\
\hline 24 & $\begin{array}{l}\text { Einschätzung von Motiven } \\
\text { der Engagierten }\end{array}$ & 4er-Skala (s.o.); halboffen & $\begin{array}{l}\text { F: Solidarität, Nr. } 51 \text { (o.S.) } \\
\text { (modifiziert) } \\
\text { A: modifiziert; } \\
\text { A: Türken: S. } 221 \text { | } \\
\text { FWS, S. } 419 \text { I } \\
\text { Motive: S. } 36 \text { f. } \\
\text { (alle modifiziert) }\end{array}$ \\
\hline 25 & $\begin{array}{l}\text { Entwicklung der Zahlen von } \\
\text { Mitgliedern, Hauptamtlichen, } \\
\text { Engagierten in den letzten } \\
5 \text { Jahren (seit 2013) }\end{array}$ & $\begin{array}{l}\text { 6er-Skala (stark gesunken; } \\
\text { gesunken; unverändert; } \\
\text { gestiegen; stark gestiegen; } \\
\text { haben wir nicht); geschlos- } \\
\text { sen }\end{array}$ & $\begin{array}{l}\text { F: ZiviZ, Nr. } 37, \text { S. } 93 \\
\text { (modifiziert) } \\
\text { A: modifiziert }\end{array}$ \\
\hline
\end{tabular}




\section{Themenblock 4: Ziele, Selbstverständnis, internes Miteinander}

Organisationssoziologische Definitionen des Terminus „Organisation“ verweisen auf das Charakteristikum der Zielgerichtetheit bzw. Zweckbezogenheit von Organisationen, die mit dem Anliegen, bestimmte Ziele zu realisieren, gegründet wurden (II. 4.3.1). Bei der Vorgabe von insgesamt elf konkreten Zielen wurden migrationsspezifische Interessen ${ }^{6}$, Geselligkeits- und Allgemeinwohlorientierungen, die Zwecke der religiösen Glaubenspraxis und der Unterstützung von schwachen Mitgliedern sowie das Anliegen (politischer) Interessenvertretung berücksichtigt. In Betracht gezogen wurden damit Aufnahmeland- und Herkunftsland- sowie Binnenund Außenorientierungen (Tab. 6.1).

Die Formulierung der Ziele erfolgte größtenteils in Eigenregie der Autorin; zudem wurden ursprünglich auf der Individualebene abgefragte, mit einem persönlichen Engagement verknüpfte migrationsspezifische Erwartungen (Motive) in kollektive Ziele der MSO umgemünzt, modifiziert und erweitert (Halm/Sauer 2005: 140 ff.; Sauer 2011: 230 ff.; II. 4.3.3.2). Als Organisationsziele formuliert können sie auch als Indikatoren für Motive der Mitglieder und Engagierten, sich in den Organisationen einzubringen, fungieren.

Die anschließende Frage nach dem Selbstverständnis der Organisation wurde - wie auch acht der insgesamt 13 gelisteten Antwortvorgaben - aus dem ZiviZSurvey 2012 übernommen. Als Items wurden bewusst auch seitens der Politik kommunizierte Bezeichnungen der MSO als „Brückenbauer und Kulturdolmetscher" oder "Integrationspromotoren“ aufgenommen. Ihr normativ aufgeladener Gehalt kann die Tendenz zu sozial erwünschtem Antwortverhalten provozieren, da soziale Anerkennung gewonnen bzw. soziale Ablehnung vermieden werden soll (Strack 1994: 14). Da die Befragung anonym und vertraulich gehalten war, war andererseits auch davon auszugehen, dass das Problem der sozialen Erwünschtheit reduziert und den tatsächlich vertretenen Selbstbildern entsprechend geantwortet wurde. Unabhängig von dem im Rahmen von Erhebungen grundsätzlich vorhandenen Problem sozial erwünschter Beantwortungen, war von Interesse und sollte überprüft werden, inwiefern die MSO an sie herangetragene Zuschreibungen und Funktionen befürworten. Die Überprüfung, inwieweit sie positiven Selbstdarstellungen gerecht werden, erfolgte im Rahmen der Auswertung mit einer separaten, breiten Inblicknahme ihrer Performanzen - insbesondere ihrer Aktivitäten und Ziele (7.2).

6 „Die Lebenssituation und Teilhabe von Zugewanderten/Landsleuten in Deutschland verbessern“; „Die eigene Herkunftssprache und -kultur in Deutschland aufrechterhalten und pflegen“, „Kontakte in die alte Heimat aufrechterhalten“, „Die Erfahrung von Ausgrenzung und Vertreibung im Herkunftsland in Deutschland bewältigen“, „Unterstützung von im Herkunftsland vertretenen politischen Positionen in Deutschland“. 
Mit der Einschätzung von Aussagen zum Miteinander in der Organisation wurde ebenfalls an eine im Erhebungsbogen des ZiviZ-Surveys 2012 enthaltene Frage angeknüpft. Sie sollte zum einen der vertieften Ermittlung des Selbstverständnisses, aber in stark komplexitätsreduzierender Weise auch der Sondierung der formalen bzw. informellen Organisationsstrukturen dienen.

Mit acht Items fokussiert wurden interne Gegebenheiten und Abläufe, konkret die Priorität eines guten Einvernehmens und solidarischen Verhaltens im Allgemeinen und - mit einem separaten Item - aufgrund eines geteilten Gottesglaubens im Besonderen. Des Weiteren war die Bedeutung von demokratischen Strukturen und Geschlechtergleichstellung sowie die Existenz von Konflikten insgesamt und zwischen unterschiedlichen Mitgliedergenerationen im Speziellen einzuschätzen. Ebenfalls beantwortet werden sollte, inwieweit ein Austausch mit Organisationen im Ausland stattfindet und ob die Zusammenarbeit zwischen Hauptamtlichen und Freiwilligen ohne Probleme verläuft.

\begin{tabular}{|c|c|c|c|}
\hline \multicolumn{4}{|c|}{ Themenblock 4: Ziele, Selbstverständnis, internes Miteinander } \\
\hline Nr. & Frage & Form der Frage & $\begin{array}{l}\text { Orientierung der Fragen }(F) / \\
\text { Antwortvorgaben }(A) \\
\text { an anderen Studien } \\
\text { (Nr./Seite) }\end{array}$ \\
\hline 26 & Ziele der MSO & $\begin{array}{l}\text { Mehrfachnennung; } \\
\text { 4er-Skala } \\
\text { (Trifft voll und ganz zu ... } \\
\text { Trifft überhaupt nicht zu); } \\
\text { halboffen }\end{array}$ & $\begin{array}{l}\text { A: Türken, S. } 221 \\
\text { (modifiziert) }\end{array}$ \\
\hline 27 & $\begin{array}{l}\text { Selbstverständnis der Orga- } \\
\text { nisation }\end{array}$ & 4er-Skala (s. o.); halboffen & $\begin{array}{l}\text { F: ZiviZ, Nr. 21, S. } 90 \\
\text { (übernommen) } \\
\text { A: modifiziert }\end{array}$ \\
\hline 28 & $\begin{array}{l}\text { Aussagen zum sozialen Mit- } \\
\text { einander in der Organisation }\end{array}$ & $\begin{array}{l}\text { 4er-Skala (s. o.); } \\
\text { geschlossen }\end{array}$ & $\begin{array}{l}\text { F: ZiviZ, Nr. 36, S. } 92 \\
\text { (modifiziert) } \\
\text { A: modifiziert }\end{array}$ \\
\hline
\end{tabular}

\section{Themenblock 5: Aktivitäten und Dienstleistungen}

Eine besonders wichtige stellte die anschließende Frage nach den Schwerpunktbereichen der Aktivitäten und Dienstleistungen der MSO dar. Da angesichts der in dieser Arbeit vertretenen breiten Definition von MSO von einem vielfältigen und sehr heterogenen Spektrum an Aktivitäten auszugehen war, wurde mit insgesamt 21 Items versucht, die potenziellen Aktivitäten möglichst erschöpfend abzubilden. Orientiert wurde sich dabei an den im ZiviZ-Survey 2012 sowie im Freiwilligensurvey 2014 vorgenommenen Differenzierungen. Die Aktivitäten 
lassen sich migrationsspezifischen, (sozial-)pädagogischen, erzieherischen, kulturellen, religiösen, geselligen, aufnahmeland- bzw. herkunftslandorientierten sowie nach innen und außen gerichteten Orientierungen zuordnen. Mit der Abfrage von Tätigkeiten verknüpft war die im Fragebogen bewusst nicht ersichtlich gemachte Kategorisierung einzelner Variablen als Indikatoren für Formen und Aktivitäten des Brückenschlags zu anderen Gruppen (bridging) einerseits sowie als Indizien für Selbstbezüglichkeit und Exklusivität der MSO (bonding) andererseits (II. 4.2.3). Diese sollten in einem separaten Analyseteil zu den beiden prominenten Ausprägungsformen des Sozialkapitals - bridging und bonding - näher untersucht und zueinander ins Verhältnis gesetzt werden (7.2).

Die Übernahme konkreter Aktivitäten in der Flüchtlingshilfe wurde separat abgefragt. Der Filterfrage, ob bestimmte Tätigkeiten ausgeführt werden (nein/ja), schloss sich die Abfrage von 17 spezifischen Aktivitäten an. Bei der Zusammenstellung der Itembatterie wurden Einzelangaben aus der EFA-Studie 2 verwendet.

Da die sogenannte ,Flüchtlingskrise“ im Jahr 2015 mit Blick auf die (politische) Anerkennung von MSO als wichtige zivilgesellschaftliche Akteure als ein markanter Entwicklungspunkt einzustufen ist, dieser Bewertung bislang aber der Charakter anekdotischer Evidenz zukommt und sie empirisch zu prüfen ist, sollte sie mit einer weiteren Frage fokussiert werden. Daher waren in einer nächsten Frage vier konkrete Aussagen zu in den MSO zu registrierenden Veränderungen einzuschätzen, die in Verbindung mit der Flucht vieler Menschen seit 2015 nach Deutschland stehen. ${ }^{7}$

Mit der nächsten Frage sollte die mit dem Aktivitätsspektrum einhergehende Zielgruppenorientierung der MSO und damit anhand eines weiteren Aspekts implizit die Offenheit der Organisationen ermittelt werden. Von den vier gelisteten Antwortmöglichkeiten (inkl. ,Sonstiges“) sollte nur eine markiert werden. ${ }^{8}$

Mit einer weiteren Frage sollte festgestellt werden, in welchem Umfeld die MSO aktiv sind; von Interesse war der Radius bzw. die Kombinationen von insgesamt sieben vorgegebenen Wirkungsbereichen, von denen mehrere markiert werden konnten: ,,im Stadtteil“, ,,in der Stadt“, ,,in der Region (Kreis/Bezirk)“, ,,in Nordrhein-Westfalen“, ,in ganz Deutschland“, ,in ganz Europa“, „International“.

\footnotetext{
${ }^{7}$ Die vier einzustufenden Aussagen lauteten wie folgt: „Unsere Organisation ist zur Anlaufstelle für geflüchtete Menschen geworden“; ,Unsere Arbeit erfährt seitens der Stadt und Politik eine höhere Aufmerksamkeit und Wertschätzung“; ,Die Zusammenarbeit mit städtischen Ämtern und Behörden hat sich intensiviert“; ,Unsere Organisation profitiert von politischen Fördermaßnahmen in der Flüchtlingshilfe“.

${ }^{8}$ Antwortvorgaben (Einfachnennung): „Unsere Aktivitäten und Angebote richten sich nur an Mitglieder“; ,Unsere Aktivitäten und Angebote richten sich an Personen mit den gleichen religiösen Überzeugungen“; ,Unsere Aktivitäten und Angebote sind für alle offen“; ,,Sonstiges, und zwar: __.
} 
Themenblock 5: Aktivitäten/Dienstleistungen und Zielgruppen

\begin{tabular}{|c|c|c|c|}
\hline Nr. & Frage & Form der Frage & $\begin{array}{l}\text { Orientierung der Fragen }(\mathrm{F}) \text { / } \\
\text { Antwortvorgaben }(\mathrm{A}) \\
\text { an anderen Studien } \\
\text { (Nr./Seite) }\end{array}$ \\
\hline 29 & $\begin{array}{l}\text { Schwerpunktbereiche } \\
\text { der Dienstleistungen und } \\
\text { Aktivitäten }\end{array}$ & $\begin{array}{l}\text { Mehrfachnennung; } \\
\text { halboffen }\end{array}$ & $\begin{array}{l}\text { F: ZiviZ, Nr. 10, S. } 87 \\
\text { (+ Ausdifferenzierung } \\
\text { S. 22f.) | FWS, S. } 89 \\
\text { F \& A: alle modifiziert }\end{array}$ \\
\hline 30 & $\begin{array}{l}\text { Ob Tätigkeiten in der Flücht- } \\
\text { lingshilfe }\end{array}$ & $\begin{array}{l}\text { Einfachnennung (nein/ja); } \\
\text { geschlossen; } \\
\text { Filter (nein: } \rightarrow \text { Frage } 33 \text { ) }\end{array}$ & - \\
\hline 31 & $\begin{array}{l}\text { Konkrete Tätigkeiten in der } \\
\text { Flüchtlingshilfe }\end{array}$ & $\begin{array}{l}\text { Mehrfachnennung, } \\
\text { halboffen }\end{array}$ & $\begin{array}{l}\text { A: EFA 2, S. } 26 \\
\text { (modifiziert) }\end{array}$ \\
\hline 32 & $\begin{array}{l}\text { Aussagen zu Veränderungen } \\
\text { in der MSO seit Flucht- } \\
\text { dynamik } 2015\end{array}$ & $\begin{array}{l}\text { 4er-Skala (Trifft voll und } \\
\text { ganz zu ... Trifft überhaupt } \\
\text { nicht zu); geschlossen }\end{array}$ & - \\
\hline 33 & $\begin{array}{l}\text { Zielgruppen der Dienst- } \\
\text { leistungen und Angebote }\end{array}$ & Einfachnennung; halboffen & $\begin{array}{l}\text { F: ZiviZ, Nr. 16, S. } 89 \\
\text { (übernommen) } \\
\text { A: modifiziert }\end{array}$ \\
\hline 34 & $\begin{array}{l}\text { Umfeld, in dem Organisation } \\
\text { aktiv ist }\end{array}$ & $\begin{array}{l}\text { Mehrfachnennung; } \\
\text { geschlossen }\end{array}$ & $\begin{array}{l}\text { F: ZiviZ, Nr. 19, S. } 89 \\
\text { (übernommen) } \\
\text { A: modifiziert }\end{array}$ \\
\hline
\end{tabular}

\section{Themenblock 6: Kooperations- und Umweltbeziehungen}

Die Beziehungen zu und Austauschverhältnisse mit anderen (Kollektiv-)Akteuren wurden v. a. über die Kooperation mit anderen Organisationen, die damit verfolgten Zielsetzungen und die im Rahmen der Zusammenarbeit wahrgenommenen Schwierigkeiten abgefragt. Die Frage, ob die Organisation zurzeit mit anderen Organisationen zusammenarbeitet oder dieses in den letzten fünf Jahren getan hat, war als Filter konzipiert (nein/ja). Bei Verneinung waren die vier folgenden Fragen zu überspringen. Bei Bejahung sollte in der anschließenden Frage hinsichtlich 13 aufgeführter Items bei möglichen Mehrfachantworten markiert bzw. ergänzt werden, mit welchen bestimmten Typen von Organisationen zusammengearbeitet wird oder wurde. Festgestellt wurde dadurch also nicht die genaue Anzahl der Kooperationspartner insgesamt, sondern die Anzahl der Organisationstypen, mit denen zusammengearbeitet wird.

Vermittels der Einstufung von neun vorgegebenen Kooperationszielen sollten mit der Beantwortung der nächsten Frage Schwerpunktsetzungen identifiziert bzw. festgestellt werden, inwieweit monetäre Anreize, das Knüpfen und Pflegen langfristiger Kontakte, der Austausch von Informationen, der Image- und Legitimitätsgewinn, die Vorteilsverschaffung gegenüber anderen Organisationen oder das Anliegen der Ausdehnung der Arbeit der eigenen MSO als jeweils wichtig(er) erachtet werden. 
Ob im Rahmen der Kooperationsarbeit bestimmte Schwierigkeiten bestehen, wurde anhand einer darauffolgenden Filterfrage ermittelt (nein/ja). Bei ihrer Bejahung sollten diese Schwierigkeiten im weiteren Verlauf konkretisiert werden. Anhand der Beantwortung von neun Items sollte herausgefunden werden, inwieweit Probleme auf asymmetrische Beziehungen, Voreingenommenheiten, Rivalität, mangelndes Vertrauen, verbale Kommunikationsschwierigkeiten oder Missverständnisse zwischen den Partnern zurückgeführt und inwieweit bürokratische und konzeptuelle Probleme bei der Durchführung von Projekten geltend gemacht werden.

Die abschließende anhand von sieben Aussagen vorzunehmende Beurteilung der Arbeit und Situation der eigenen Organisation sollte zum einen Raum für auf die geleistete Arbeit bezogene Selbstbeurteilungen geben („Unsere Arbeit müsste professioneller und qualifizierter sein"). Zum anderen sollten Einschätzungen zur Zufriedenheit mit der städtischen Einbindung und zum Wissen über Informationsund Förderkulturen ermittelt werden (,Wir wissen, wo und wie wir Fördermittel beantragen können"). Ferner war es ein Anliegen, Urteile zu gegenüber anderen MSO ggf. bestehenden Konkurrenzverhältnissen sowie öffentlichen Anerkennungsprozessen zu eruieren (,Unsere Arbeit erfährt zu wenig öffentliche Wertschätzung und Anerkennung“). Die beiden Aussagen „Unsere Organisation ist in starkem Ausmaß mit Fremdenfeindlichkeit und Diskriminierung konfrontiert“ und „Die öffentliche Meinung über unsere Gruppe ist negativ" waren als Variablen auch im Rahmen von an dieser Stelle nicht zu vertiefenden Hypothesenprüfungen bedeutsam. Denn die Art der Wahrnehmung öffentlicher Diskurse durch die MSO hat Auswirkungen auf ihr Verhalten gegenüber anderen Organisationen - oder anders formuliert: Handeln und Auftreten der MSO hängen auch von ihrer Wahrnehmung und Behandlung durch die gesellschaftliche Umwelt ab (Pries 2013a: 5; Pries 2010a: 20).

Die in Bezug auf die Arbeit und Situation der MSO getätigten Angaben sind aufschlussreich hinsichtlich der Registrierung und notwendigen Bearbeitung von organisations- und umweltbezogenen Problemlagen und sie implizieren Mitteilungen von Handlungs- und Unterstützungsbedarfen. Die Artikulation solcher Bedarfe wurde auch durch den ganz am Ende des Fragebogens eingeräumten Platz für Kommentare und Anmerkungen ermöglicht, wobei diese Option auch zur Teilnahme an der Befragung motivieren sollte. 
Themenblock 6: Kooperations- und Umweltbeziehungen

Zusammenarbeit mit anderen Organisationen

\begin{tabular}{|c|c|c|c|}
\hline Nr. & Frage & Form der Frage & $\begin{array}{l}\text { Orientierung der Fragen }(\mathrm{F}) / \\
\text { Antwortvorgaben }(\mathrm{A}) \\
\text { an anderen Studien } \\
\text { (Nr./Seite) }\end{array}$ \\
\hline 35 & $\begin{array}{l}\text { Ob Zusammenarbeit mit } \\
\text { anderen Organisationen } \\
\text { (zurzeit oder in den letzten } \\
5 \text { Jahren) }\end{array}$ & $\begin{array}{l}\text { Einfachnennung (nein/ja); } \\
\text { geschlossen; } \\
\text { Filter (nein: } \rightarrow \text { Frage 40) }\end{array}$ & - \\
\hline 36 & $\begin{array}{l}\text { Organisationen als Koopera- } \\
\text { tionspartner }\end{array}$ & $\begin{array}{l}\text { Mehrfachnennung; } \\
\text { halboffen }\end{array}$ & $\begin{array}{l}\text { F: ZiviZ, Nr. 12, S. 88 I } \\
\text { Solidarität, Nr. } 31 \text { (o.S.) } \\
\text { (modifiziert) } \\
\text { A: modifiziert }\end{array}$ \\
\hline 37 & Ziele der Kooperationen & $\begin{array}{l}\text { 4er-Skala (Trifft voll und } \\
\text { ganz zu ... Trifft überhaupt } \\
\text { nicht zu); halboffen }\end{array}$ & $\begin{array}{l}\text { F: ZiviZ, Nr. 13, S. } 88 \\
\text { (modifiziert) } \\
\text { A: modifiziert }\end{array}$ \\
\hline 38 & $\begin{array}{l}\text { Ob Schwierigkeiten innerhalb } \\
\text { der Kooperationsarbeit }\end{array}$ & $\begin{array}{l}\text { Einfachnennung (nein/ja); } \\
\text { geschlossen; } \\
\text { Filter (nein: } \rightarrow \text { Frage 40) }\end{array}$ & $\begin{array}{l}\text { F: Solidarität, Nr. } 33 \text { (o. S.) } \\
\text { MDO, Nr. VII. 2, S. } 93 \\
\text { (modifiziert) } \\
\text { A: modifiziert }\end{array}$ \\
\hline 39 & Art der Schwierigkeiten & $\begin{array}{l}\text { Mehrfachnennung; } \\
\text { geschlossen }\end{array}$ & - \\
\hline \multicolumn{4}{|c|}{ Arbeit und Situation der MSO } \\
\hline 40 & $\begin{array}{l}\text { Aussagen zur Arbeit und } \\
\text { Situation der MSO }\end{array}$ & $\begin{array}{l}\text { 4er-Skala (s.o.); } \\
\text { geschlossen }\end{array}$ & $\begin{array}{l}\text { F: ZiviZ, Nr. 52, S. } 95 \\
\text { (modifiziert) } \\
\text { A: modifiziert }\end{array}$ \\
\hline 41 & $\begin{array}{l}\text { Kommentare oder Anmer- } \\
\text { kungen }\end{array}$ & offen & - \\
\hline
\end{tabular}

\subsection{Anmerkungen zum Auswertungsverfahren}

\section{Vorgehensweise}

In den folgenden Unterkapiteln und Abschnitten werden die Ergebnisse für das gesamte Sample $(n=257)$ berichtet. Dies erfolgt inhaltlich aspektorientiert entlang der für den Auswertungsteil vorgegebenen Gliederung. Dabei erweist sich eine blockweise Vorgehensweise als synergetisch und opportun: Die Darstellung der einzelnen thematisch verorteten Befunde wird jeweils direkt zusammen mit statistischen Analyseverfahren (Tests) sowie mit der Überprüfung von Hypothesen geleistet. Aufgrund der Integration der Hypothesen in den Auswertungsteil werden sie in einer inhaltlich passenden Reihenfolge abgearbeitet. Darüber hinaus erfolgt die Schilderung jeweiliger Ergebnisse im Auswertungsteil (7.1) jeweils 
direkt zusammen mit einer Interpretation und Einordnung der Befunde in den Stand von Theorie und Forschung. Eine solche Verortung ausschließlich und erst in einem die Gesamtauswertung abschließenden Kapitel - in Loslösung von den konkreten Ergebnissen - zu leisten, wäre zu unübersichtlich und würde die Nachvollziehbarkeit nicht gewährleisten. Innerhalb des langen Auswertungsteils werden wesentliche Ergebnisse - dem besseren Überblick halber - in typografisch hervorgehobenen „sprechenden Überschriften“ kondensiert.

Für die Auswertung der Daten wurde SPSS 25 verwendet. In einem ersten Schritt werden im Rahmen der deskriptiven Analyse der Daten jeweils deren Verteilung über die verschiedenen Antwortmöglichkeiten geschildert. Aufgrund fehlender Werte variieren die zur Analyse herangezogenen Fallzahlen (n). Um den Fließtext nicht mit zu vielen Zahlen zu überlasten, wird auf die Wiedergabe der einzelnen Zahlen (n) zumeist verzichtet. Dies gilt auch für den im Untersuchungsfokus stehenden Vergleich zwischen den beiden Gruppen der religiösen und nicht-religiösen bzw. säkularen Vereinigungen (Abk.: $\mathrm{n}_{\mathbf{R}}$ und $\mathrm{n}_{\mathbf{S}}$ ). Zur Unterscheidung der beiden Organisationskategorien werden in der Ergebnisdarstellung auch die Kürzel „RMO“ (für religiöse) und „MO“ (für nicht-religiöse Vereinigungen) verwendet.

Für die Ergebnispräsentation im Fließtext wird die im Fragebogen häufiger vorgegebene vierstufige Antwortskala (,,Trifft voll und ganz zu“ .., ,Trifft überhaupt nicht $\mathrm{zu}^{\text {“) }}$ zwecks einer komprimierten Darstellung von Zustimmungsraten in zwei Antwortkategorien zusammengefasst ${ }^{9}$ und nach den für die Stufen ,trifft

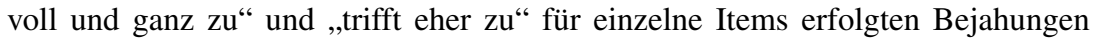
zumeist in absteigender Folge berichtet. Die der Veranschaulichung dienenden Balkendiagramme stellen die Prozentwerte hingegen jeweils detailliert für alle vier Skalenausprägungen und nach dem Wert einer kompletten Zustimmung (,trifft voll und ganz zu“) in absteigender Sortierung dar.

Für die Interpretation und Erörterung der Ergebnisse werden durchgängig für religiöse und nicht-religiöse MSO zu identifizierende Auffälligkeiten bzw. Unterschiede berichtet. Relevante bivariate Zusammenhangsmaße (SpearmanKorrelationskoeffizient rho, Cramers $V$ ), Differenzen in zentralen Tendenzen ( $U$-Test), Effektstärken (Cohen's $d$ und $r$ ) und Signifikanzniveaus $(p)^{10}$ sind im Fließtext vermerkt oder in separaten Tabellen ausgewiesen. Berichtet werden

\footnotetext{
${ }^{9}$ Zusammenfassung zu den zwei Kategorien ,Trifft voll und ganz zu/Trifft eher zu“ und „Trifft eher nicht zu/Trifft überhaupt nicht zu“.

${ }^{10}$ Die Signifikanz beschreibt die Wahrscheinlichkeit, dass es sich bei den Testergebnissen nicht um einen Zufall handeln kann bzw. die statistische Irrtumswahrscheinlichkeit und damit die Belastbarkeit der Ergebnisse. Die Signifikanzniveaus $p$ sind wie folgt festgelegt: $* * * \leq 0,001[0,1 \%]|* * \leq 0,01[1 \%]| * \leq 0,05[5 \%]$.
} 
i. d. R. nur signifikante Testergebnisse. In Abschnitt 7.3 werden die wesentlichen Ergebnisse der Auswertung zusammengetragen.

\section{Zentrale statistische Analyseverfahren}

Mann-Whitney-U-Test

Ein im Rahmen der Auswertung häufig angewandter statistischer Test ist der Mann-Whitney-U-Test (kurz: $U$-Test). Mit ihm kann überprüft werden, ob sich die Messwerte von zwei Stichproben bzw. Gruppen signifikant unterscheiden. Es handelt sich um ein nicht-parametrisches, recht voraussetzungsfreies, für unabhängige Stichproben verwendbares Verfahren und ein Äquivalent zum voraussetzungsreicheren $\boldsymbol{t}$-Test. ${ }^{11}$ Mit dem $U$-Test kann über den gemittelten Rang von zwei Gruppen (d. h. die durch die Gruppengröße dividierte Rangsumme) und auf Basis der asymptotischen Signifikanz $\left(n_{S}+n_{R}>30\right)$ überprüft werden, ob sich die zentralen Tendenzen der religiösen und nicht-religiösen MSO ähneln oder derart voneinander unterscheiden, dass von zwei separaten Grundgesamtheiten ausgegangen werden kann (Brosius 2006: 851). Aufgrund der inversen Codierung (Vierer-Ordinalskala Code 1 bis 4) im Fragebogen zeigen niedrigere mittlere Ränge und tiefere Werte für die Gruppenmediane (Abk.: $\mathrm{Md}_{\mathrm{S}}$ und $\mathrm{Md}_{\mathrm{R}}$ ) im Rahmen dieser Auswertung für den $U$-Test zumeist eine stärkere Ausprägung des Merkmals bei einer jeweiligen Gruppe an. ${ }^{12}$

\section{Zusammenhangsmaße bzw. Effektstärken}

Zusammen mit dem $U$-Test wird die Effektstärke $r$ berechnet. Werte für $r$ von über 0,1 sind als schwacher Effekt, von über 0,3 als mittelstarker Effekt und Werte größer

${ }^{11}$ Mit dem $\boldsymbol{t}$-Test kann bei Vorliegen einer intervallskalierten abhängigen Variablen für zwei unabhängige Stichproben geprüft werden, ob sich deren Mittelwerte signifikant voneinander unterscheiden (Brosius 2018: 557 ff.). Der $U$-Test kann mit ordinalskalierten Variablen, bei einer nicht vorhandenen Normalverteilung der Daten und auch mit Ausreißern und kleinen Stichproben berechnet werden.

Alternativ zum $U$-Test könnte für die Schätzung eines Zusammenhangs zwischen einer natürlichen dichotomen Variablen, die zwei Gruppen definiert, sowie einer ordinalskalierten Variablen, auch jeweils die Biseriale Rangkorrelation angewendet werden, die dem $U$-Test entspricht (Wirtz 2020). Die für den $U$-Test und die Biseriale Rangkorrelation berechneten Effekt- bzw. Zusammenhangsstärken sind folglich identisch.

${ }^{12}$ Bei ordinalskalierten Variablen, die im Rahmen der Erhebung häufig verwendet wurden (Vierer-Skala: $1=$ Trifft voll und ganz zu; $2=$ Trifft eher zu; $3=$ Trifft eher nicht $z u$; $4=$ Trifft überhaupt nicht zu), sind die Median- oder Zentralwerte zu berichten, d. h. die genau in der Mitte einer Datenverteilung liegenden Werte. Diese können bei signifikanten Testergebnissen, die unterschiedliche Verteilungen der beiden Gruppen anzeigen, auch den gleichen Wert annehmen. 
als 0,5 als starker Effekt zu interpretieren (Cohen 1988: 79 ff.). Zur Prüfung von linearen Zusammenhängen zwischen zwei mindestens ordinalskalierten Variablen wird auf den Rangkorrelationskoeffizienten nach Spearman (rho) zurückgegriffen (Cleff 2012: 113 ff.). Als schwache, mittlere bzw. starke Zusammenhänge gelten betragsmäßige Korrelationen von rho über 0,1, über 0,3 bzw. über 0,5 (ebd.). Beim $t$-Test wird für die Messung der Effektstärke hingegen auf das Maß Cohen's $\boldsymbol{d}$ zurückgegriffen. Den Faustregeln nach Cohen (1992: 157) zufolge sind Ergebnisse für Cohen's $d$ ab 0,2 als ein kleiner Effekt, ab 0,5 als ein mittlerer und ab 0,8 als ein großer Effekt zu interpretieren. ${ }^{13}$

Cramers $\boldsymbol{V}$ ist ein bivariates Zusammenhangsmaß, das bei nominalskalierten Variablen anzuwenden ist. Cramers $V$ kann nur positive Werte zwischen 0 (kein Zusammenhang, statistische Unabhängigkeit) und 1 (perfekter Zusammenhang, vollständige Determination) annehmen; daher kann keine Aussage über die Richtung des Zusammenhangs gemacht werden. Für die Interpretation von Cramers $V$ sind daher Kreuztabellen heranzuziehen. In der Fachliteratur besteht Uneinigkeit darüber, ab welchen Werten bei Cramers $V$ von einem ,schwachen“, ,mittleren“ oder ,starken“ Zusammenhang gesprochen wird. ${ }^{14}$ Im Rahmen dieser Arbeit werden folgende Bewertungsmaßstäbe zugrunde gelegt:

, $\mathrm{V} \in[0,00 ; 0,10[\rightarrow$ kein Zusammenhang; $\mathrm{V} \in[0,10 ; 0,30[\rightarrow$ schwacher Zusammenhang; $\mathrm{V} \in[0,30 ; 0,60[\rightarrow$ mittlerer Zusammenhang; $\mathrm{V} \in[0,60 ; 1,00] \rightarrow$ starker Zusammenhang“(Cleff 2012: 92).

\section{Explorative Faktorenanalyse (EFA)}

Die Explorative Faktorenanalyse (kurz: EFA) ist ein strukturentdeckendes multivariates Analyseverfahren. Dieses ist für die Auswertung opportun, da keine gesicherten Annahmen über bestehende Zusammenhänge zwischen den Variablen existieren. Das statistische Verfahren dient der Strukturierung und Reduzierung von Daten und erleichtert deren Interpretation. Die Faktorenanalyse errechnet übergeordnete Hintergrundgrößen (Faktoren), die möglichst unabhängig voneinander und aussagekräftig die latente Verwandtschaft bzw. Beziehungsstruktur von

\footnotetext{
${ }^{13}$ Die Berechnung der Effektstärken erfolgte mit dem Online-Rechner Psychometrica: https:// www.psychometrica.de/effektstaerke.html.

Für eine bessere Lesbarkeit werden die im Rahmen des $U$-Tests berechneten Teststatistiken ( $U$ - und $z$-Werte) sowie die für Effektstärken bzw. Zusammenhangsmaße ermittelten Zahlen auf zwei Nachkommastellen gerundet.

${ }^{14}$ Einige Autoren betonen, dass die Interpretation der Effektgröße kontextabhängig und eine automatisierte Anwendung der Cohen-Richtlinien (Cohen 1988) daher problematisch sei (Sun 2008: 12). Stattdessen solle eine Einschätzung der Zusammenhangsstärke auf Basis von Erfahrungswerten zu vergleichbaren inhaltlichen Fällen und Tabellen erfolgen (Brosius 2006: 430). Letztere sind für den hier relevanten Forschungsgegenstand jedoch nicht verfügbar.
} 
vielen miteinander hoch korrelierenden Variablen aufdeckt, letztere bündelt und von miteinander weniger in Wechselbeziehung stehenden Variablen separiert. Der praktische Hintergrund ist, dass bestimmte Antwortvorgaben gehäuft zusammen markiert wurden, während das bei anderen nicht der Fall war und sie sich daher quasi abstoßen.

Auf Basis einer rotierten Faktorladungsmatrix kann die Zuordnung einer Variablen zu einem Faktor erfolgen. Im Idealfall lassen sich alle Variablen eines berücksichtigten Sets auf jeweils einen Faktor zurückführen. Die Berechnung von Faktorladungen einer Variablen (zwischen -1 und +1) verdeutlicht den Grad der Korrelation zwischen der Variablen und dem Faktor. Absolutwerte unter 0,5 sollten unterdrückt werden (Brosius 2006: 783), und wenn eine Variable auf mehreren Faktoren eine Ladung von $\geq 0,5$ aufweist, ist sie bei allen betreffenden Faktoren zur Interpretation heranzuziehen (Backhaus et al. 2018: 399).

\section{Multiple lineare Regression}

Bei der multiplen linearen Regression handelt es sich um ein strukturprüfendes multivariates Analyseverfahren, mit dem getestet wird, ob zwischen einer abhängigen und mehreren unabhängigen Variablen ein Zusammenhang besteht (Backhaus et al. 2018: 57-118). Dabei unterliegt die lineare Regression diversen Modellprämissen. Hierbei handelt es sich um den Ausschluss von Autokorrelation, um Homoskedastizität (Varianzhomogenität) und um Normalverteiltheit der Fehlerwerte. Die Überprüfung, ob die Fehlerterme autokorreliert sind, erfolgt mittels des Durbin-Watson-Tests. Hierbei sollte die Teststatistik zwischen 1 und 3 liegen, damit von unkorrelierten Fehlertermen ausgegangen werden kann (Field 2013:311). Die Annahme homoskedastischer Fehler wird mittels eines Residualplots überprüft. Hierbei sollte die Streuung über die Punktwolke gleichmäßig sein, damit von gleichen Varianzen ausgegangen werden kann (Backhaus et al. 2018: 94 f.). Des Weiteren wird die Normalverteilungsannahme der Fehlerterme über ein QuantilQuantil-Plot (Q-Q-Plot) der Residuen untersucht (Hartung et al. 2005: 847 ff.). Bei den Residuen handelt es sich um die Abweichungen zwischen den geschätzten Werten der abhängigen Variablen und deren tatsächlichen Beobachtungen. Sie dienen somit als „Schätzer“ für die theoretischen Modellfehler. In einem Q-Q-Plot werden dabei die Quantile der Residuen gegen die Quantile einer Normalverteilung abgetragen (ebd.: 34 f.). Damit von einer Normalverteiltheit ausgegangen werden kann, sollten die Punkte in der Grafik annähernd auf einer Geraden streuen.

Neben den zuvor dargestellten Annahmen in einem linearen Modell kann bei der multiplen linearen Regression im Speziellen das Problem der Multikollinearität auftreten. Multikollinearität entsteht häufig, wenn mathematische Abhängigkeiten zwischen den unabhängigen Variablen vorliegen. Unter Multikollinearität sind die 
verwendeten Schätzmethoden nicht mehr in der Lage, den Effekt einer unabhängigen Variablen auf die abhängige Variable richtig zuzuordnen. Zur Überprüfung auf Multikollinearität werden die Varianzinflationsfaktoren (VIF) für jede unabhängige Variable bestimmt. Hierbei sollten diese Kennzahlen kleiner 10 sein, damit von keinem Multikollinearitätsproblem ausgegangen werden muss (Fahrmeir et al. 2009: 170). Zur Beurteilung eines linearen Modells kann das Bestimmtheitsmaß $\left(\mathrm{R}^{2}\right)$ verwendet werden. Hierbei handelt es sich um den Anteil an Varianz der abhängigen Variablen, welcher durch die unabhängigen Variablen erklärt wird. Das gewöhnliche Bestimmtheitsmaß hat eine zentrale Schwäche: Mit Hinzunahme auch irrelevanter Variablen steigt es automatisch an. Deswegen wird in dieser Arbeit das korrigierte Bestimmtheitsmaß (korrigiertes $\mathrm{R}^{2}$ ) verwendet, welches diese Schwäche nicht besitzt (ebd.: 160 f.).

Weiterhin können einzelne Fälle stark von den übrigen abweichen und die geschätzte Regressionsgerade hierdurch beeinflussen. In diesem Zusammenhang wird von einflussreichen Fällen (,Ausreißern“) gesprochen. Mittels der Cook's Distanz können einflussreiche Fälle identifiziert werden. Hierbei sollte die Cook's Distanz nicht größer 1 sein, da dieser Fall ansonsten als sehr beeinflussend zu betrachten und aus der Analyse zu entfernen ist; Beobachtungen größer 0,5 gelten als auffällig und sollten näher betrachtet werden (Fahrmeir et al. 2009: 178).

Open Access Dieses Kapitel wird unter der Creative Commons Namensnennung 4.0 International Lizenz (http://creativecommons.org/licenses/by/4.0/deed.de) veröffentlicht, welche die Nutzung, Vervielfältigung, Bearbeitung, Verbreitung und Wiedergabe in jeglichem Medium und Format erlaubt, sofern Sie den/die ursprünglichen Autor(en) und die Quelle ordnungsgemäß nennen, einen Link zur Creative Commons Lizenz beifügen und angeben, ob Änderungen vorgenommen wurden.

Die in diesem Kapitel enthaltenen Bilder und sonstiges Drittmaterial unterliegen ebenfalls der genannten Creative Commons Lizenz, sofern sich aus der Abbildungslegende nichts anderes ergibt. Sofern das betreffende Material nicht unter der genannten Creative Commons Lizenz steht und die betreffende Handlung nicht nach gesetzlichen Vorschriften erlaubt ist, ist für die oben aufgeführten Weiterverwendungen des Materials die Einwilligung des jeweiligen Rechteinhabers einzuholen.

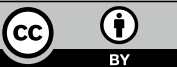

